\title{
Modeling and Evaluation of Human Motor Skills in a Virtual Tennis Task
}

\author{
Yoshiyuki Tanaka, Member, IEEE, Masataka Ishii, Toshio Tsuji, Member, IEEE, \\ and Nobuaki Imamura, Member, IEEE
}

\begin{abstract}
The present paper develops a virtual tennis system using robotic devices for the rehabilitation of upper arm movements, and discusses an evaluation method of human motor skills for natural stroke in the dynamic task. In the training, a trainee manipulates the handle of an impedance-controlled robot to hit a ball by a racket toward the center of a circular target on the wall with considerations of motion smoothness and timing. A reference hand motion for the target task is clarified through a set of training experiments with 6 health volunteers and computationally expressed in the framework of a minimum jerk model. The designed index based on the reference model will be effective to quantitatively evaluate the recovery of motor skills for natural stroke in the virtual tennis task.
\end{abstract}

\section{INTRODUCTION}

Aging society in Japan has led to increase the number of stroke patients who need to recover their motor skills, while the shorthanded situation in the rehabilitation field becomes a serious problem more than ever. Certainly, an advanced rehabilitation system using robotic devices has been expected as one of effective means for that social problem.

Many robot-assisted training systems have been developed for the upper extremity in recent years [1]-[4]. For example, Krebs et al. [1], [2] developed the training system for extremities using an impedance-controlled robot, in which the trainee manipulates an end-effector to follow a target trajectory on the feedback display. Furusho et al. [4] developed the 3-D rehabilitation system using ER actuators with highly safety and performance. These robotic systems would enable not only to repeat the exact same movements consistently based on a conventional therapy but also to provide the individual data measured during training tests and to develop a novel training method. The previous studies, however, do not clarify a reference motion for the target task that will be required in designing a quantitative index to evaluate recovery of motion functions and in teaching skillful motion to a trainee directly or indirectly.

On the other hand, dynamic properties of human movements are often expressed with mechanical impedance parameters [5], [6]. Focusing on the regulation ability of human

This research work was supported in part by a Grant-in-Aid for Scientific Research from the Japanese Ministry of Education, Science and Culture (18760193), and the 21st Century COE Program of JSPS (Japan Society for the Promotion of Science) on Hyper Human Technology toward the 21st Century Industrial Revolution.

Y. Tanaka, M. Ishii, and T. Tsuji are with Graduate School of Engineering, Hiroshima University, Higashi-hiroshima, Japan \{ytanaka, masataka, tsuji\}@bsys.hiroshima-u.ac.jp

N. Imamura is with Department of Engineering, Hiroshima Kokusai University, Higashi-hiroshima, Japan n-imamur@it.hirokokuu.ac.jp impedance properties, Tsuji and Tanaka [7]-[10] developed the virtual sports training system using the impedancecontrolled robot. They demonstrated the relationship between trainee's skill-levels and hand impedance properties estimated at the moments of motion rest in dynamical behavioral tasks, such as virtual tennis, air-hockey and curling, but did not sufficiently investigate motion timing and smoothness, which are main functions of the cerebellum, that would be enhanced by the designed training task.

In this paper, a virtual tennis system using an impedancecontrolled robot with two D.O.F is newly developed to clarify and model a reference motion in the training task through a set of training experiments with healthy volunteers. This paper is organized as follows: Section II explains a structure of the developed robotic system and a model of the virtual tennis task. Section III describes an experimental method and shows the changes in hand spatio-temporal trajectory on the learning of the training task. Finally, the motor skills enhanced in the training are evaluated by using the index based on a reference motion expressed with a minimum jerk model in Section IV.

\section{VIRTUAL TENNIS SYSTEM}

\section{A. Structure}

Fig. 1 shows an overview of the virtual curing system. The system is composed of an impedance-controlled robot [11] for providing virtual force to a trainee, a DSP instrument for robot control and signal processing, and a biofeedback display for presenting virtual curing game.

The robot is composed of two linear motor tables with one degree of freedom ( $x$ axis: Nihon Thompson Coop., maximum force \pm 10 [kgf]; $y$ axis: Nihon Seikou Coop., maximum force \pm 40 [kgf]) that are placed orthogonally in order to carry out the two-dimensional hand motion exercise. Hand force generated by the trainee is measured by a sixaxis force/torque sensor (BL Autotec Co. Ltd., resolution: force $x$ and $y$ axes, $0.05[\mathrm{~N}] ; z$ axis, $0.15[\mathrm{~N}]$; torque, 0.003 $[\mathrm{Nm}])$ attached on the handle of robot. The handle position is measured by an encoder built in the linear motor tables (resolution: $x$ and $y$ axes, $1.0[\mu \mathrm{m}])$. The DSP instrument (A\&D Co. Ltd., AD5410) realizes the precise impedancecontrol of robot motion in real-time and for estimating human impedance properties during a virtual curling task. The feedback display presents the training information in the operation. 


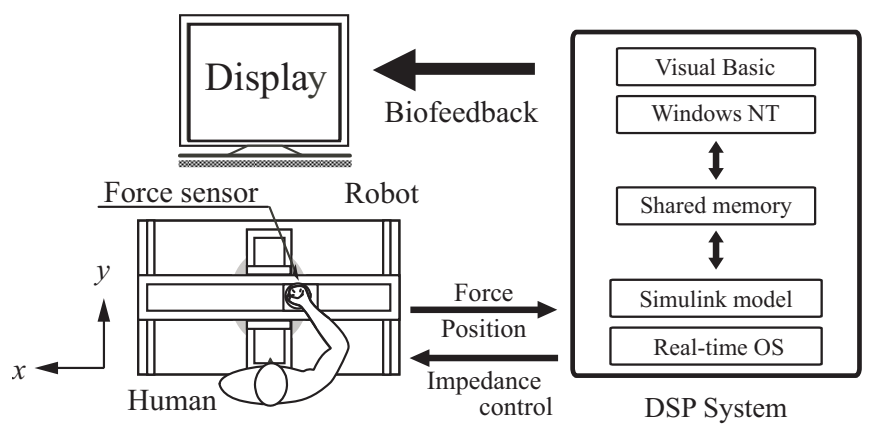

(a)

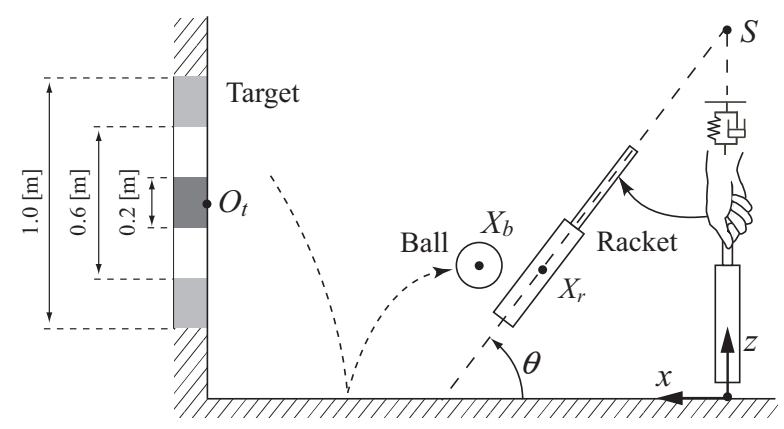

(b)

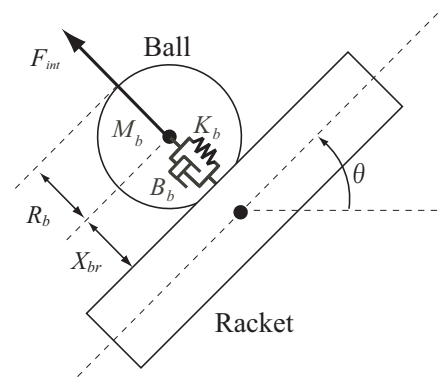

(c)

Fig. 1. An overview of the virtual tennis task.

\section{B. Virtual Tennis Task}

Fig. 1(b) illustrates a virtual tennis model installed into the experimental system. A virtual racket is rigid and rotates around the point $S$, while mechanical properties of a virtual ball including a racket strings are expressed with a viscoelastic model (Fig. 1(c)).

Dynamics of the impedance-controlled handle during a task is given by

$$
F_{e}=M_{r} \ddot{X}_{e}+B_{r} \dot{X}_{e}
$$

where $F_{e} \in \Re^{2}$ is the hand force, $X_{e} \in \Re^{2}$ is the hand position, $M_{r}=\operatorname{diag} .\left(m_{r}, m_{r}\right) \in \Re^{2 \times 2}$ and $B_{r}=$ $\operatorname{diag} .\left(b_{r}, b_{r}\right) \in \Re^{2 \times 2}$ are robot inertia and viscosity, respectively. The racket angle $\theta$ is given by

$$
\theta=\frac{\pi}{2}-\frac{5 \pi}{4} x_{e}
$$

where $x_{e}$ is the hand position on the $x$-axis.

The ball is thrown with a certain initial velocity and its

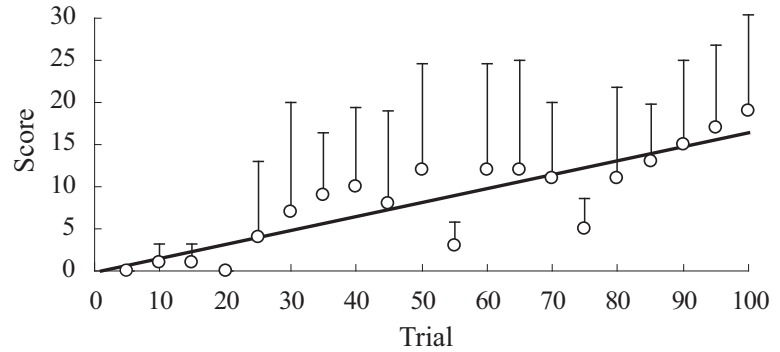

(a)

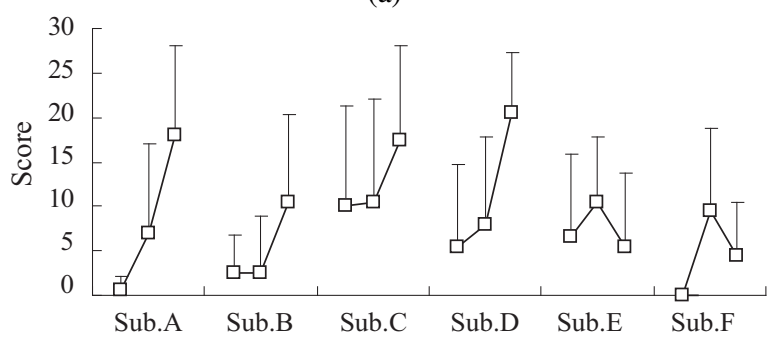

(b)

Fig. 2. Changes of the task performance for the trial number.

motion is calculated by

$$
M_{b}\left(\ddot{X}_{b}+g\right)+B_{a i r} \dot{X}_{b}=F_{i n t}
$$

where $X_{b} \in \Re^{2}$ is the ball position, $g$ is the gravitational acceleration, $F_{\text {int }} \in \Re^{2}$ is the interaction force between ball and racket, the $M_{b}=$ diag. $\left(m_{b}, m_{b}\right) \in \Re^{2 \times 2}$ and $B_{\text {air }}=$ diag. $\left(b_{a}, b_{a}\right) \in \Re^{2 \times 2}$ are the ball inertia and air resistance, respectively. Just during the contact between ball and racket, $F_{\text {int }}\left(0 \leq\left|X_{b r}\right| \leq R_{b}\right)$ is given by

$$
F_{\text {int }}=R(\theta)^{\mathrm{T}} B_{b r} R(\theta) \dot{X}_{b r}+R(\theta)^{\mathrm{T}} K_{b r} R(\theta) d X_{b r}
$$

where $X_{b r} \in \Re^{2}$ is the vector normal to the racket surface, $R(\theta) \in \Re^{2 \times 2}$ is the rotational matrix transforming from the basic coordinate system to the local coordinate system at the racket, $d X_{b r} \in \Re^{2}$ is the deformation of the ball

$$
d X_{b r}=R_{b} \frac{X_{b r}}{\left|X_{b r}\right|}-X_{b r} .
$$

A trainee is asked to strike an approaching ball with a racket in the virtual tennis space (the $x-z$ plane) by manipulating a handle attached on the moving part of the robot, while the DSP system simulates dynamic behaviors of ball and racket at the same time.

\section{TRAINING EXPERIMENT}

\section{A. Method}

Training experiments were carried out with 6 healthy volunteers in this paper. The ball was thrown from the initial position $X_{b}(0)=(4.0,1.0)^{\mathrm{T}}[\mathrm{m}]$ with the initial velocity $\dot{X}_{b}(0)=(-3.0,2.0)^{\mathrm{T}}[\mathrm{m} / \mathrm{s}]$, the initial angle of racket was set at $\theta(0)=\pi / 2$ [rad], and the center of the target circle was at $O_{t}=(5.0,1.0)^{\mathrm{T}}[\mathrm{m}]$. The other experimental parameters were set as $\left(m_{b}, b_{b r}, k_{b r}\right)=(0.05[\mathrm{~kg}], 2.0[\mathrm{Ns} / \mathrm{m}], 500$ $[\mathrm{N} / \mathrm{m}]), R_{b}=0.05[\mathrm{~m}], b_{a}=0.01[\mathrm{Ns} / \mathrm{m}], m_{r}=5.0[\mathrm{~kg}], b_{r}$ $=5.0[\mathrm{Ns} / \mathrm{m}]$. 

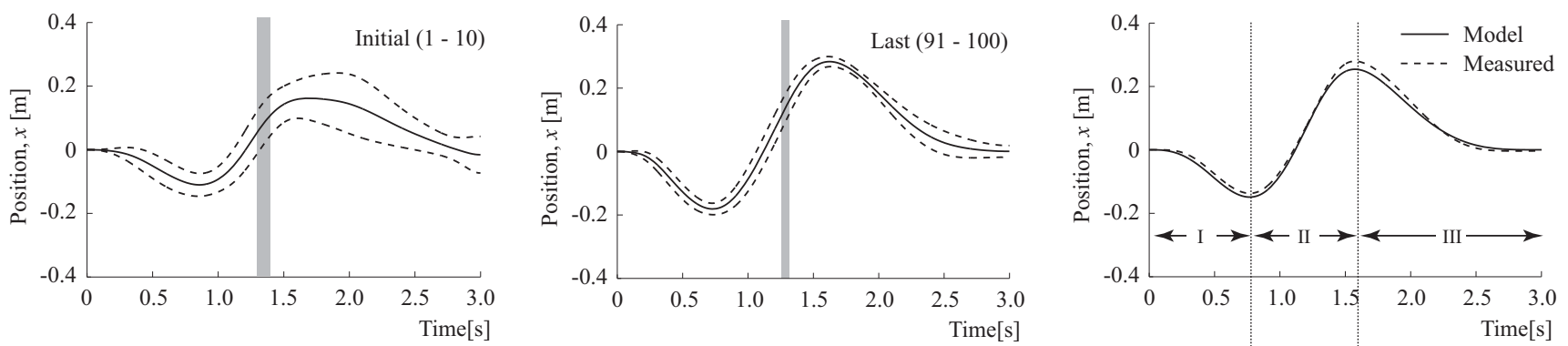

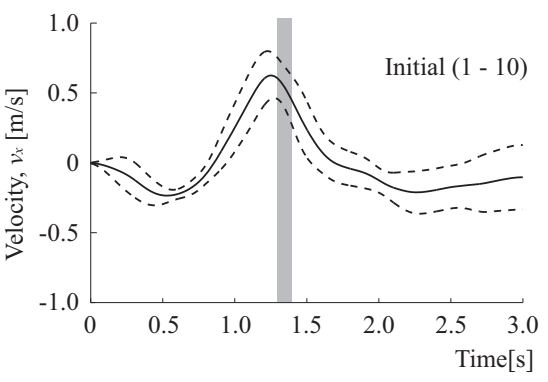

(a)

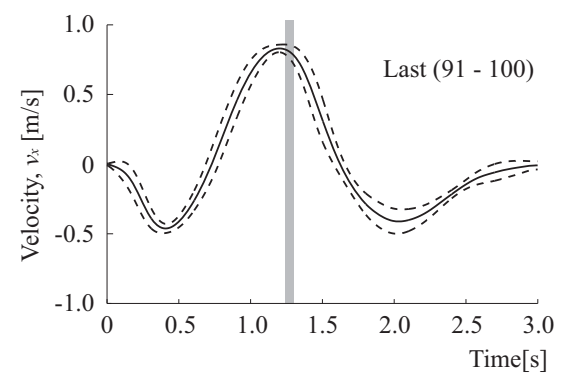

(b)

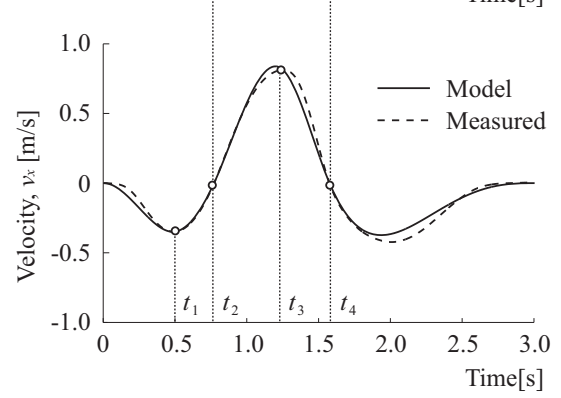

(c)

Fig. 3. Measured and simulated hand spatio-temporal trajectories in the virtual tennis task.

A subject stood in front of the system, and he was asked to strike the ball to directly hit the center of a target circle settled on the wall after brief explanations on the virtual tennis task and the operating system (Fig. 1(a)). The task performance was scored according to the distance between target center and ball-stroked position on the wall as 30 points within $0.1[\mathrm{~m}], 20$ points within $0.3[\mathrm{~m}], 10$ points within 0.5 [m], and 5 points over 0.5 [m] (Fig. 1 (b)). No point recorded when the ball indirectly hit the wall with contacts with the ground. 100 trials were set for each subject to investigate the changes of hand movements in the process of skill-acquisition.

\section{B. Results}

Fig. 2(a) shows the trial history of a mean score in each five trials for Sub. A. The result demonstrates that the subject became more skillful in getting a higher point in proportional to the trial number. The figure (b) shows the average scores of the initial, middle, and last 10 trials for all subjects. In the task operations, Subs. A-C took a preparatory hand motion to the reverse direction from the initial hand position before hitting the ball while Subs. D-F did not. All subjects in the former group improved their scores much more than the latter group. This suggests that the preparatory motion will be effective to record higher points constantly. Therefore, we focused on motor behaviors with the preparatory motion in the target task.

Figs. 3 (a) and (b) show the time histories of hand spatiotemporal trajectories along the $x$-direction for Sub. A in the first ten trials (Fig. 3(a)) and the last ten trials (Fig. 3(b)), where the initial time was the ball-throwing time and the solid line is the average trajectory. The shade zones represent the terms of contact between ball and racket. The hand trajectories generated by the subject converged to the average trajectory after enough trials, and the shade zone was narrow.
The skilled subject plans to hit the ball at the regular period for getting higher points by generating a unique and smooth spatio-temporal trajectory. Note that the peak of a velocity profile appeared just before the ball was hitted. This suggests that a human involuntary maximizes his hand velocity at hitting a moving object. The similar tendency was observed on all subjects, regardless of the preparatory motion.

\section{SKill EVAluation}

\section{A. Modeling of Reference Motion}

To evaluate motor skills in the virtual tennis task, the average of hand trajectories along the $x$-direction for 30point scored in the last ten trials were extracted as a reference motion (the dotted line in Fig. 3(c)). Since the reference motion was regarded as the combination of three point-to-point motions, we utilized a minimum jerk model [12], a wellknown computational model for reaching movements with a bell-shaped velocity profile, with the evaluation function $J$ given by

$$
J=\frac{1}{2} \int_{0}^{t_{f}}\left(\frac{d^{3} x_{e}}{d t^{3}}\right)^{2} d t
$$

where $t_{f}$ is the terminal time of hand motion.

The simulated reference motion was plotted as the solid line in Fig. 3(c), where the velocities measured at $t_{i}(i=$ $1,2, \ldots, 4)$ were specified as the constraints in minimizing the value of the function $J . t_{3}$ is of the time at hitting the ball. It can be found that the simulated motion is almost agreed with the average one. This suggests that a skilled subject generates his hand trajectory for the dynamic task enhanced motion smoothness and timing in the framework of the minimum jerk model. 


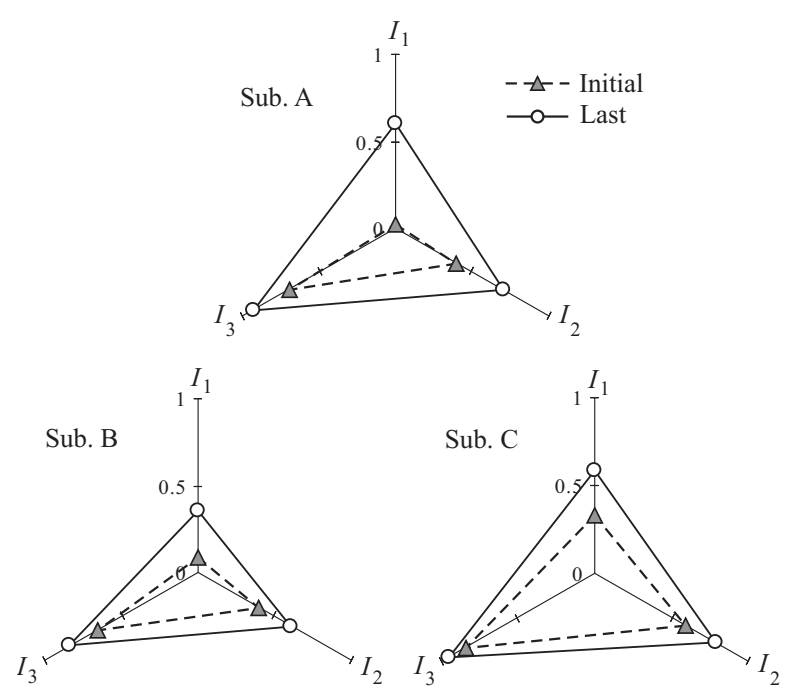

(a)

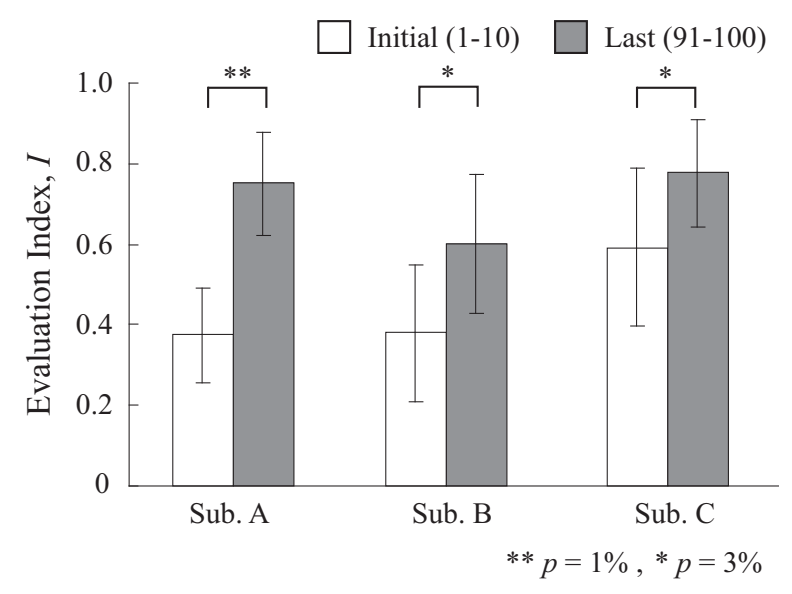

(b)

Fig. 4. Evaluation of the task performances before and after training.

\section{B. Evaluation Results}

A quantitative evaluation index $I$ was designed using the reference model as

$$
I=w_{1} \frac{p}{30}+w_{2}\left(1-\frac{e}{e_{\max }}\right)+w_{3} r^{2}
$$

where $p$ is the point obtained, $e$ and $r^{2}$ are the difference between reference and measured velocity profiles and the determine of coefficient, respectively, $e_{\max }$ is the summing integral value of the rectified reference velocity profile, and $0 \leq w_{i} \leq 1(i=1,2,3)$ is the weight coefficient. The first term $\left(I_{1}\right)$ is for the evaluation of task performance, and the rest terms $\left(I_{2}, I_{3}\right)$ are of motor skills requiring in the virtual tennis task, such as motion smoothness and timing. The best evaluation is at $I=w_{1}+w_{2}+w_{3}$.

Fig. 4 (a) shows the mean value of each term with $w_{i}=1$ for the initial and last ten trials by Subs. A-C, where the best evaluation is at $I_{i}=1$. The triangle for the last trials is obviously larger than one for the initial trials. Finally, Fig. 4 (b) shows the total evaluation results with $w_{i}=1 / 3$ for Subs.
A-C. It can be found that the subjects significantly improved their motor skills by the repetition. These results demonstrate that the designed index shows which and how much motor skills in the virtual tennis are improved quantitatively.

\section{CONCLUSIONS}

This paper discussed the evaluation method of motor skill in the virtual tennis task in which a trainee must control his arm movements with consideration of timing. The training experiments shown that the skilled subjects scored higher points while generating the almost same spatiotemporal trajectory. The skilled hand motion was regarded as one of a reference trajectory in the target task, and was successfully expressed in the framework of the minimum jerk model with constraints. Finally, the evaluation index based on the reference model was validated through applying to the experimental data before and after enough trials. The future research will be directed to improve an evaluation index and to do the training test with the corporation of stroke patients.

\section{ACKNOWLEDGMENT}

The authors would like to appreciate the kind supports of the A\&D Co. Ltd. on the DSP instrument.

\section{REFERENCES}

[1] H. I. Krebs, N. Hogan, M. L. Aisen and B. T. Volpe: "Robot-aided neurorehabilitation," IEEE Transactions on Rehabilitaion Engineering, Vol. 6, No. 1, pp. 75-87, 1998.

[2] H. I. Krebs, B. T. Volpe, M. L. Aisen and N. Hogan: "Increasing productivity and quality of care: Robot-aided neuro rehabilitation," Jurnal of Rehabilitation Reserch and Development, Vol.37, No.6, pp. 639-652, 2000.

[3] Y. Okajima, N. Tanaka, M. Hasegawa, N. Uchida, A. Kimura, Y. Tomita, T. Horiuchi, M. Kondo, T. Sakaki: "Therapeutic Exercise Machine: Soft Motion by the Impedance Control Mechanism," Sogo Rehabilitation, Vol. 26, No. 4, pp. 363-369, Igaku-shoin Ltd., 1998. (In Japanese)

[4] J. Furusho, K. Koyanagi, K. Nakanishi, U. Ryu, S. Takenaka, A. Inoue, K. Domen, and K. Miyakoshi: "Development of A 3-D Rehabilitation System for Upper Limbs Using ER Actuators in a NEDO Project," International Journal of Modern Physics B, Vol. 19, No. 7-9, pp.15911597, 2005.

[5] F. A. Mussa-Ivaldi, N. Hogan and E. Bizzi: "Neural, mechanical and geometric factors subserving arm in humans," Journal of Neuroscience, Vol. 5, No. 10, pp. 2732-2743, 1985.

[6] T. Tsuji, P. G. Morasso, K. Goto, K. Ito: "Human hand impedance characteristics during maintained posture," Biological Cybernetics, Vol. 72, pp. 457-485, 1994.

[7] T. Tsuji, Y. Sumida, M. Kaneko, S.Kawamura: "A Virtual Sports System for Skill Training," Journal of Robotics and Mechatronics, Vol. 13, No. 2, pp. 168-175, 2001.

[8] Y. Tanaka, T. Tsuji, and M. Kaneko: "A Virtual Air Hockey System for Skill Training," Proc. of the 1st International Conference on Information Technology in Mechatronics, pp. 136-141, 2001.

[9] T. Tsuji, Y. Takeda and Y. Tanaka: "Analysis of Mechanical Impedance in Human Arm Movements using a Virtual Tennis System," Biological Cybernetics, Vol. 91, No. 5, pp. 295-305, 2004.

[10] Y. Tanaka, K. Matsushita, T. Tsuji: "Sensorimotor characteristics in human arm movements during a virtual curling task," Transactions of the Society of Instrument and Control Engineers, Vol. 42, 12, 12881294, 2006 (in Japanese).

[11] N. Hogan: "Impedance Control: An approach to Manipulation, Parts I, II, III," ASME Journal of Dynamic Systems, Measurement, and Control, Vol. 107, No. 1, pp. 1-24, 1985.

[12] T. Flash, N. Hogan : The Coordination of Arm Movements: An Experimentally Confirmed Mathematical Model, The Journal of Neuroscience, Vol.5, No.7, 1688-1703, 1985. 